\title{
ON QUADRATURE CONVERGENCE OF EXTENDED LAGRANGE INTERPOLATION
}

\author{
WALTER GAUTSCHI AND SHIKANG LI
}

\begin{abstract}
Quadrature convergence of the extended Lagrange interpolant $L_{2 n+1} f$ for any continuous function $f$ is studied, where the interpolation nodes are the $n$ zeros $\tau_{i}$ of an orthogonal polynomial of degree $n$ and the $n+1$ zeros $\hat{\tau}_{j}$ of the corresponding "induced" orthogonal polynomial of degree $n+1$. It is found that, unlike convergence in the mean, quadrature convergence does hold for all four Chebyshev weight functions. This is shown by establishing the positivity of the underlying quadrature rule, whose weights are obtained explicitly. Necessary and sufficient conditions for positivity are also obtained in cases where the nodes $\tau_{i}$ and $\hat{\tau}_{j}$ interlace, and the conditions are checked numerically for the Jacobi weight function with parameters $\alpha$ and $\beta$. It is conjectured, in this case, that quadrature convergence holds for $|\alpha| \leq \frac{1}{2},|\beta| \leq \frac{1}{2}$.
\end{abstract}

\section{INTRODUCTION}

If $\pi_{n}(\cdot ; w), n \geq 1$, denotes the $n$ th-degree orthogonal polynomial on $[-1,1]$ with respect to a positive weight function $w$, and $\left(L_{n} f\right)(\cdot)$ the Lagrange interpolation polynomial of degree $<n$ interpolating $f$ at the zeros $\left\{\tau_{i}\right\}$ of $\pi_{n}$, it is a well-known result of Erdös and Turán [2] that $L_{n} f$ converges in the mean to $f$ for any continuous function $f$. That is,

$$
\lim _{n \rightarrow \infty}\left\|f-L_{n} f\right\|_{w}=0, \text { all } f \in C[-1,1],
$$

where $\|g\|_{w}=\left(\int_{-1}^{1} g^{2}(t) w(t) d t\right)^{1 / 2}$. Attempts have been made in the past to obtain an analogous result for the extended Lagrange interpolant $\left(\hat{L}_{2 n+1} f\right)(\cdot)$ interpolating $f$ at $2 n+1$ points - the $n$ points $\left\{\tau_{i}\right\}$ and $n+1$ additional points $\left\{\hat{\tau}_{j}\right\}$ suitably chosen. A particularly interesting choice of the $\hat{\tau}_{j}$, first suggested by Bellen [1], is given by the zeros of $\hat{\pi}_{n+1}$, the polynomial $\hat{\pi}_{n+1}(\cdot)=\pi_{n+1}\left(\cdot ; \pi_{n}^{2} w\right)$ of degree $n+1$ "induced by $\pi_{n}$ ", i.e., orthogonal relative to the weight function $\pi_{n}^{2} w$ (cf. [5]). Concrete results have only been obtained in the case of Chebyshev weight functions. The one of the second kind, $w(t)=\left(1-t^{2}\right)^{1 / 2}$, is particularly easy, since in this case $\left\{\tau_{i}\right\} \cup\left\{\hat{\tau}_{j}\right\}$ are precisely the zeros of $U_{n} T_{n+1}=U_{2 n+1}$ (cf. [1]), and one is led back to the Erdös-Turán result. For all other three Chebyshev weight functions, however, one of us [3] has shown that mean convergence cannot hold for all continuous functions.

Received by the editor April 20,1995

1991 Mathematics Subject Classification. Primary 41A05, 65D32; Secondary 33C45.

(C)1996 American Mathematical Society 
It may be interesting to ask the same question for what Erdös and Turán called quadrature convergence. In their scenario, that would mean

$$
\lim _{n \rightarrow \infty} \int_{-1}^{1}\left[f(t)-\left(L_{n} f\right)(t)\right] w(t) d t=0, \text { all } f \in C[-1,1],
$$

which is obviously true, since the integral over $L_{n} f$ is just the $n$-point Gauss quadrature sum relative to the weight function $w$. Is it true that the same holds for extended interpolation,

$$
\lim _{n \rightarrow \infty} \int_{-1}^{1}\left[f(t)-\left(\hat{L}_{2 n+1} f\right)(t)\right] w(t) d t=0, \text { all } f \in C[-1,1] ?
$$

The answer is yes, if the underlying quadrature rule has all weights positive, as follows from a classical result of Pólya [6]. We will show in $\S 2$ of this note that this is indeed the case, for all four Chebyshev weight functions, and in the process also determine explicitly the weights of the quadrature rules involved. Moreover, it will be shown in $\S 3$ that positivity also holds if the nodes $\tau_{i}$ and $\hat{\tau}_{j}$ interlace, provided the Gauss weights for the weight function $w$ satisfy certain inequalities. The latter are checked numerically for the Jacobi weight function $w^{(\alpha, \beta)}(t)=(1-t)^{\alpha}(1+t)^{\beta}$, and evidence is produced suggesting that the quadrature weights in question are indeed positive if $|\alpha| \leq \frac{1}{2},|\beta| \leq \frac{1}{2}$.

One could be tempted to take the zeros of $\pi_{n+1}$ as the additional nodes $\hat{\tau}_{j}$ since interlacing is then guaranteed. However, the quadrature rule implied by (1.3) is then simply the $(n+1)$-point Gaussian rule for $w$ (all nodes $\tau_{i}$ receive weight zero), and we are back again to the Erdös-Turán result!

\section{Chebyshev weight functions}

The weights of the interpolatory quadrature rule implied by (1.3) are given by

$$
\begin{aligned}
& \lambda_{i}=\int_{-1}^{1} \frac{\pi_{n}(t) \hat{\pi}_{n+1}(t)}{\left(t-\tau_{i}\right) \pi_{n}^{\prime}\left(\tau_{i}\right) \hat{\pi}_{n+1}\left(\tau_{i}\right)} w(t) d t, \quad i=1,2, \ldots, n ; \\
& \mu_{j}=\int_{-1}^{1} \frac{\pi_{n}(t) \hat{\pi}_{n+1}(t)}{\left(t-\hat{\tau}_{j}\right) \pi_{n}\left(\hat{\tau}_{j}\right) \hat{\pi}_{n+1}^{\prime}\left(\hat{\tau}_{j}\right)} w(t) d t, \quad j=1,2, \ldots, n+1,
\end{aligned}
$$

where $\pi_{n}(\cdot)=\pi_{n}(\cdot ; w)$ and $\hat{\pi}_{n+1}(\cdot)=\pi_{n+1}\left(\cdot ; \pi_{n}^{2} w\right)$. The rule has degree of exactness equal to $2 n$. For reasons indicated in the Introduction, it suffices to look at Chebyshev weights of the first, third, and fourth kind.

2.1. Chebyshev weight of the first kind. Here the weight function is $w_{1}(t)=$ $\left(1-t^{2}\right)^{-1 / 2}$, and $\pi_{n}$ is the Chebyshev polynomial of the first kind,

$$
\pi_{n}(t)=T_{n}(t), \quad T_{n}(\cos \theta)=\cos n \theta,
$$

whereas $\hat{\pi}_{n+1}$ is given by [3]

$$
\hat{\pi}_{n+1}(t)=T_{n+1}(t)-\frac{1}{2} T_{n-1}(t), \quad n \geq 1 .
$$

Theorem 2.1. For $w_{1}(t)=\left(1-t^{2}\right)^{-1 / 2}$, the quadrature weights $\lambda_{i}$ and $\mu_{j}$ in (2.1), (2.2) are given by

$$
\lambda_{i}=\frac{\pi}{3 n}, \quad i=1,2, \ldots, n ;
$$




$$
\mu_{j}=\frac{2 \pi}{3} \frac{1}{n+\frac{3}{9-8 \hat{\tau}_{j}^{2}}}, \quad j=1,2, \ldots, n+1,
$$

where $\hat{\tau}_{j}$ are the zeros of $\hat{\pi}_{n+1}$. All weights are positive.

Proof. It follows easily from (2.3) and (2.4) that $\pi_{n}^{\prime}\left(\tau_{i}\right)=n(-1)^{i-1} / \sin \theta_{i}$ and $\hat{\pi}_{n+1}\left(\tau_{i}\right)=\frac{3}{2}(-1)^{i} \sin \theta_{i}$, where $\theta_{i}=(2 i-1) \pi / 2 n$, so that

$$
\pi_{n}^{\prime}\left(\tau_{i}\right) \hat{\pi}_{n+1}\left(\tau_{i}\right)=-\frac{3}{2} n \text {. }
$$

It remains, for $\lambda_{i}$, to evaluate the integral

$$
\int_{-1}^{1} \frac{T_{n}(t)\left[T_{n+1}(t)-\frac{1}{2} T_{n-1}(t)\right]}{t-\tau_{i}} w_{1}(t) d t .
$$

Since $\tau_{i}$ is a zero of $T_{n}$, the integral, by orthogonality of the $T_{m}$, reduces to

$$
-\frac{1}{2} \int_{-1}^{1} \frac{T_{n}(t)}{t-\tau_{i}} T_{n-1}(t) w_{1}(t) d t
$$

which in turn is equal to $-\frac{\pi}{2}$. This follows by observing, if $n>1$, that

$$
\frac{T_{n}(t)}{t-\tau_{i}}=2 T_{n-1}(t)+\text { lower-degree terms, }
$$

by orthogonality, and by using

$$
\int_{-1}^{1} T_{m}^{2}(t) w_{1}(t) d t=\frac{\pi}{2}, \quad m \geq 1
$$

For $n=1$, the reasoning is the same except for the factor and divisor 2 in the last two formulae, which must be replaced by 1 . The result (2.5) now follows immediately.

To evaluate the constant in the denominator of $(2.2)$, we let

$$
\hat{\tau}_{j}=\cos \hat{\theta}_{j}
$$

and obtain from

$$
\hat{\pi}_{n+1}(\cos \theta)=\cos (n+1) \theta-\frac{1}{2} \cos (n-1) \theta
$$

by differentiation and the addition formula for the sine

$$
\hat{\pi}_{n+1}^{\prime}\left(\hat{\tau}_{j}\right)=\frac{1}{2 \sin \hat{\theta}_{j}}\left\{(n+3) \sin n \hat{\theta}_{j} \cos \hat{\theta}_{j}+(3 n+1) \cos n \hat{\theta}_{j} \sin \hat{\theta}_{j}\right\} .
$$

Since

$$
\cos (n+1) \hat{\theta}_{j}-\frac{1}{2} \cos (n-1) \hat{\theta}_{j}=0,
$$

and using here the addition formula for the cosine, we find

$$
\sin n \hat{\theta}_{j}=\frac{1}{3} \frac{\cos n \hat{\theta}_{j} \cos \hat{\theta}_{j}}{\sin \hat{\theta}_{j}} .
$$

Together with (2.8), this yields after a simple computation

$$
\pi_{n}\left(\hat{\tau}_{j}\right) \hat{\pi}_{n+1}^{\prime}\left(\hat{\tau}_{j}\right)=\frac{1}{2} T_{n}^{2}\left(\hat{\tau}_{j}\right)\left\{\frac{n+3}{3} \frac{\hat{\tau}_{j}^{2}}{1-\hat{\tau}_{j}^{2}}+3 n+1\right\} .
$$


It is known from [3, Eq. (2.7)] that $T_{n}^{2}(t)=9\left(1-t^{2}\right) /\left(9-8 t^{2}\right)$ for $t=\hat{\tau}_{j}$. Therefore,

$$
\pi_{n}\left(\hat{\tau}_{j}\right) \hat{\pi}_{n+1}^{\prime}\left(\hat{\tau}_{j}\right)=\frac{3\left(9 n+3-8 n \hat{\tau}_{j}^{2}\right)}{2\left(9-8 \hat{\tau}_{j}^{2}\right)}=\frac{3}{2}\left(n+\frac{3}{9-8 \hat{\tau}_{j}^{2}}\right) .
$$

For the integral in (2.2), we proceed as follows:

$$
\int_{-1}^{1} \frac{T_{n}(t)\left[T_{n+1}(t)-\frac{1}{2} T_{n-1}(t)\right]}{t-\hat{\tau}_{j}} w_{1}(t) d t=2 \int_{-1}^{1} T_{n}^{2}(t) w_{1}(t) d t=\pi
$$

The first equality is a result of the orthogonality of the $T_{m}$ and the fact that

$$
\frac{T_{n+1}(t)-\frac{1}{2} T_{n-1}(t)}{t-\hat{\tau}_{j}}=2 T_{n}(t)+\text { lower-degree terms. }
$$

Combining (2.10) and (2.9) yields (2.6).

The positivity of the quadrature weights is an immediate consequence of $-1<$ $\hat{\tau}_{j}<1$ for the $\mu_{j}$, and trivial for the $\lambda_{i}$.

Since $\sum_{i=1}^{n} \lambda_{i}+\sum_{j=1}^{n+1} \mu_{j}=\pi$, it follows from Theorem 2.1 that the nodes $\hat{\tau}_{j}$ must satisfy

$$
\sum_{j=1}^{n+1} \frac{1}{n+\frac{3}{9-8 \hat{\tau}_{j}^{2}}}=1
$$

2.2. Chebyshev weights of the third and fourth kind. Because of the remark at the beginning of $\$ 3.2$ below, it suffices to examine the Chebyshev weight function of the third kind, $w_{3}(t)=(1-t)^{-1 / 2}(1+t)^{1 / 2}$, for which the relevant polynomials are

$$
\pi_{n}(t)=V_{n}(t), \quad V_{n}(\cos \theta)=\frac{\cos \left(n+\frac{1}{2}\right) \theta}{\cos \frac{1}{2} \theta}
$$

and $[3$, Eq. (2.17)]

$$
\hat{\pi}_{n+1}(t)=T_{n+1}(t)-\frac{1}{2} T_{n}(t), \quad n \geq 1 .
$$

Theorem 2.2. For $w_{3}(t)=(1-t)^{-1 / 2}(1+t)^{1 / 2}$, the quadrature weights $\lambda_{i}$ and $\mu_{j}$ in (2.1), (2.2) are given by

$$
\begin{gathered}
\lambda_{i}=\frac{2 \pi}{3} \frac{1+\tau_{i}}{2 n+1}, \quad i=1,2, \ldots, n ; \\
\mu_{j}=\frac{2 \pi}{3} \frac{1+\hat{\tau}_{j}}{n+\frac{4-2 \hat{\tau}_{j}}{5-4 \hat{\tau}_{j}}}, \quad j=1,2, \ldots, n+1,
\end{gathered}
$$

where $\tau_{i}$ and $\hat{\tau}_{j}$ are the zeros of $\pi_{n}$ and $\hat{\pi}_{n+1}$, respectively. All weights are positive.

Proof. From (2.12) and (2.13), one obtains by an elementary computation that $\pi_{n}^{\prime}\left(\tau_{i}\right)=\left(n+\frac{1}{2}\right)(-1)^{i-1} /\left(\cos \frac{1}{2} \theta_{i} \sin \theta_{i}\right)$ and $\hat{\pi}_{n+1}\left(\tau_{i}\right)=\frac{3}{2}(-1)^{i} \sin \frac{1}{2} \theta_{i}$, where $\theta_{i}=(2 i-1) \pi /(2 n+1)$, so that the constant in the integral of $(2.1)$ is

$$
\pi_{n}^{\prime}\left(\tau_{i}\right) \hat{\pi}_{n+1}\left(\tau_{i}\right)=-\frac{3}{4} \frac{2 n+1}{1+\tau_{i}} .
$$


The integral itself is

$$
\begin{aligned}
I_{n} & =\int_{-1}^{1} \frac{V_{n}(t)}{t-\tau_{i}}\left[T_{n+1}(t)-\frac{1}{2} T_{n}(t)\right] w_{3}(t) d t \\
& =\int_{-1}^{1} \frac{V_{n}(t)}{t-\tau_{i}}(1+t)\left[T_{n+1}(t)-\frac{1}{2} T_{n}(t)\right] w_{1}(t) d t .
\end{aligned}
$$

Since, by the recurrence relation for the $T_{m}$, we have

$$
(1+t)\left[T_{n+1}(t)-\frac{1}{2} T_{n}(t)\right]=\frac{1}{2} T_{n+2}(t)+\frac{3}{4} T_{n+1}(t)-\frac{1}{4} T_{n-1}(t),
$$

we can use the orthogonality of the $T_{m}$ with respect to $w_{1}$ to simplify:

$$
I_{n}=-\frac{1}{4} \int_{-1}^{1} \frac{V_{n}(t)}{t-\tau_{i}} T_{n-1}(t) w_{1}(t) d t .
$$

Now $V_{n}(t)$ has leading coefficient $2^{n}$, if $n \geq 2$, so that

$$
I_{n}=-\int_{-1}^{1} T_{n-1}^{2}(t) w_{1}(t) d t=-\frac{\pi}{2}, \quad n \geq 2 .
$$

The same result holds also for $n=1$. Combining it with (2.16) yields (2.14).

Letting as before $\hat{\tau}_{j}=\cos \hat{\theta}_{j}$, putting $t=\cos \theta$ in (2.13), and differentiating with respect to $\theta$ gives

$$
\hat{\pi}_{n+1}^{\prime}\left(\hat{\tau}_{j}\right)=\frac{n+2}{4} \frac{\sin \left(n+\frac{1}{2}\right) \hat{\theta}_{j}}{\sin \frac{1}{2} \hat{\theta}_{j}}+\frac{3 n+2}{4} \frac{\cos \left(n+\frac{1}{2}\right) \hat{\theta}_{j}}{\cos \frac{1}{2} \hat{\theta}_{j}} .
$$

Since

$$
\cos (n+1) \hat{\theta}_{j}-\frac{1}{2} \cos n \hat{\theta}_{j}=0
$$

this simplifies to

$$
\hat{\pi}_{n+1}^{\prime}\left(\hat{\tau}_{j}\right)=\frac{V_{n}\left(\hat{\tau}_{j}\right)}{4}\left(\frac{n+2}{3} \frac{1+\hat{\tau}_{j}}{1-\hat{\tau}_{j}}+3 n+2\right) .
$$

From [3, Eq. (2.22)] it is known that

$$
V_{n}^{2}(t)=\frac{9(1-t)}{(1+t)(5-4 t)} \text { for } t=\hat{\tau}_{j},
$$

so that the constant in the integral of $(2.2)$ becomes

$$
\pi_{n}\left(\hat{\tau}_{j}\right) \hat{\tau}_{n+1}^{\prime}\left(\hat{\tau}_{j}\right)=\frac{3}{2} \frac{n+\frac{4-2 \hat{\tau}_{j}}{5-4 \hat{\tau}_{j}}}{1+\hat{\tau}_{j}} .
$$

The integral, on the other hand, is

$$
\int_{-1}^{1} \frac{T_{n+1}(t)-\frac{1}{2} T_{n}(t)}{t-\hat{\tau}_{j}} V_{n}(t) w_{3}(t) d t,
$$

which, since

$$
\frac{T_{n+1}(t)-\frac{1}{2} T_{n}(t)}{t-\hat{\tau}_{j}}=V_{n}(t)+\text { lower-degree terms },
$$

reduces to

$$
\int_{-1}^{1} V_{n}^{2}(t) w_{3}(t) d t=\pi, \quad n \geq 1 .
$$

Together with (2.17), this yields (2.15). 
The positivity of the weights is evident from (2.14), (2.15).

Analogously to (2.11) one finds, after an elementary calculation, that

$$
\sum_{j=1}^{n+1} \frac{1+\hat{\tau}_{j}}{n+\frac{4-2 \hat{\tau}_{j}}{5-4 \hat{\tau}_{j}}}=1 .
$$

\section{JACOBI WEIGHT FUNCTIONS}

For more general weight functions, in particular the Jacobi weight function $w(t)=w^{(\alpha, \beta)}(t)$, where $w^{(\alpha, \beta)}(t)=(1-t)^{\alpha}(1+t)^{\beta}$, we have only conjectural results based on numerical experimentation. We are especially interested in cases where the nodes $\left\{\tau_{i}\right\}$ and $\left\{\hat{\tau}_{j}\right\}$ interlace,

$$
\hat{\tau}_{n+1}<\tau_{n}<\hat{\tau}_{n}<\tau_{n-1}<\cdots<\hat{\tau}_{2}<\tau_{1}<\hat{\tau}_{1} .
$$

We shall assume in this section (in slight contrast to $\S 2$ ) that the polynomials $\pi_{n}$ and $\hat{\pi}_{n+1}$ are monic.

3.1. Quadrature weights for interlacing nodes. We assume, as in $\S 2$, that $n$ is given and fixed. Our computations are based on the following theorem.

Theorem 3.1. Let $w$ be any (positive) weight function for which the nodes $\left\{\tau_{i}\right\}$, $\left\{\hat{\tau}_{j}\right\}$ (defined in $\S 2$ ) interlace. Then the quadrature weights $\lambda_{i}$ and $\mu_{j}$ in (2.1), (2.2) are all positive if and only if

$$
\lambda_{i}^{G}>\frac{\left\|\pi_{n}\right\|_{w}^{2}}{\left|\pi_{n}^{\prime}\left(\tau_{i}\right) \hat{\pi}_{n+1}\left(\tau_{i}\right)\right|}, \quad i=1,2, \ldots, n,
$$

where $\lambda_{i}^{G}$ are the Christoffel numbers of the n-point Gaussian quadrature rule for the weight function $w$, and $\left\|\pi_{n}\right\|_{w}^{2}=\int_{-1}^{1} \pi_{n}^{2}(t) w(t) d t$.

Proof. We first show that the interlacing property implies $\mu_{j}>0$. It is clear from (3.1) that

$$
\pi_{n}\left(\hat{\tau}_{j}\right) \hat{\pi}_{n+1}^{\prime}\left(\hat{\tau}_{j}\right)>0, \quad j=1,2, \ldots, n+1 .
$$

Thus the constant in the denominator of (2.2) is positive. In the integral that remains, the integrand is a monic polynomial of degree $2 n$. Its $(2 n)$ th derivative divided by $(2 n)$ ! is therefore constant equal to 1 , and the $n$-point Gauss formula with remainder term yields

$$
\begin{aligned}
\int_{-1}^{1} \frac{\pi_{n}(t) \hat{\pi}_{n+1}(t)}{t-\hat{\tau}_{j}} w(t) d t= & \sum_{k=1}^{n} \lambda_{k}^{G} \frac{\pi_{n}\left(\tau_{k}\right) \hat{\pi}_{n+1}\left(\tau_{k}\right)}{\tau_{k}-\hat{\tau}_{j}} \\
& +\int_{-1}^{1} \pi_{n}^{2}(t) w(t) d t=\left\|\pi_{n}\right\|_{w}^{2}
\end{aligned}
$$

since $\pi_{n}\left(\tau_{k}\right)=0$ for all $k$. Therefore,

$$
\mu_{j}=\frac{\left\|\pi_{n}\right\|_{w}^{2}}{\pi_{n}\left(\hat{\tau}_{j}\right) \hat{\pi}_{n+1}^{\prime}\left(\tau_{j}\right)}, \quad j=1,2, \ldots, n+1,
$$

and the positivity of the $\mu_{j}$ follows from (3.3). 
Similarly, for the $\lambda_{i}$ we have

$$
\int_{-1}^{1} \frac{\pi_{n}(t)}{t-\tau_{i}} \hat{\pi}_{n+1}(t) w(t) d t=\lambda_{i}^{G} \pi_{n}^{\prime}\left(\tau_{i}\right) \hat{\pi}_{n+1}\left(\tau_{i}\right)+\left\|\pi_{n}\right\|_{w}^{2},
$$

so that from $(2.1)$

$$
\lambda_{i}=\lambda_{i}^{G}+\frac{\left\|\pi_{n}\right\|_{w}^{2}}{\pi_{n}^{\prime}\left(\tau_{i}\right) \hat{\pi}_{n+1}\left(\tau_{i}\right)}, \quad i=1,2, \ldots, n .
$$

Now, however, interlacing implies

$$
\pi_{n}^{\prime}\left(\tau_{i}\right) \hat{\pi}_{n+1}\left(\tau_{i}\right)<0, \quad i=1,2, \ldots, n,
$$

so that $\lambda_{i}>0$ for all $i$ if and only if (3.2) holds.

3.2. Numerical results for the Jacobi weight function. For $w(t)=w^{(\alpha, \beta)}(t)$ it suffices to consider $\beta \geq \alpha>-1$, since an interchange of $\alpha$ and $\beta$ only changes the sign of the argument $t$ in $\pi_{n}(t)$ and $\hat{\pi}_{n+1}(t)$, hence the signs of the zeros $\tau_{i}$ and $\hat{\tau}_{j}$, and the weights $\lambda_{i}$ and $\mu_{j}$ in (2.1), (2.2) remain the same, as is easily seen.

In order to check the positivity of the weights $\lambda_{i}$ and $\mu_{j}$ numerically, we used Theorem 3.1 and examined, first of all, whether interlacing of the zeros holds, and if so, whether or not the inequalities (3.2) are valid for all $n$ up to some large limit (below we take $n \leq 160$ ). For computational purposes we found it convenient to write these inequalities in the form

$$
\lambda_{i}^{G}>\frac{\beta_{0} \beta_{1} \cdots \beta_{n}}{\prod_{\substack{k=1 \\ k \neq i}}^{n}\left|\tau_{i}-\tau_{k}\right| \prod_{j=1}^{n+1}\left|\tau_{i}-\hat{\tau}_{j}\right|}, \quad i=1,2, \ldots, n,
$$

where the $\beta$ 's are the coefficients in the recurrence relation

$$
\begin{gathered}
\pi_{\nu+1}(t)=\left(t-\alpha_{\nu}\right) \pi_{\nu}(t)-\beta_{\nu} \pi_{\nu-1}(t), \quad \nu=0,1,2, \ldots, \\
\pi_{0}(t)=1, \pi_{-1}(t)=0
\end{gathered}
$$

for the polynomials $\pi_{\nu}(\cdot)=\pi_{\nu}\left(\cdot ; w^{(\alpha, \beta)}\right)$. To generate these coefficients, and with them the polynomial $\pi_{n}$ and its zeros $\tau_{i}$, we used the routines recur and gauss in [4]. Similarly for the polynomial $\hat{\pi}_{n+1}$, where we used the routines indp and gauss. All calculations were done in double precision on a Sun SPARCstation IPX, using Fortran Version 2.0, for $n=1(1) 160$. We found that interlacing and/or positivity fails for $\alpha<-\frac{1}{2}$ and $\alpha>1$, and also for $-\frac{1}{2} \leq \alpha \leq 1$ and $\beta>1$. On the other hand, there is strong evidence for both interlacing and positivity to hold if $|\alpha| \leq \frac{1}{2}$, $|\beta| \leq \frac{1}{2}$. Both may even hold for somewhat larger values of $\alpha$ and $\beta$, as suggested in Fig. 3.1, where they seem to hold in the triangular-like region, and its reflection with respect to the diagonal $\alpha=\beta$, bounded on the left by the line $\alpha=-\frac{1}{2}$, below by $\alpha=\beta$, and on top by the dashdotted line (for $1 \leq n \leq 40$ ), the dashed line (for $1 \leq n \leq 80$ ), and the solid line (for $1 \leq n \leq 160$ ). We say "seem to hold" since interlacing and the inequality $\left(3.2^{\prime}\right)$ were verified numerically only for discrete points in the $(\alpha, \beta)$-plane spaced apart by .1 in most of the region, and by .001 (in the $\beta$-values) near the top of the region. We also verified the failure (for some $n$ ) of either interlacing or $\left(3.2^{\prime}\right)$ for $\alpha=-\frac{1}{2}-.01$ and $\beta=-\frac{1}{2}(.1) 1$, as well as for $\alpha=\beta=1(.1) 2$. It seems safe, therefore, to state the following conjecture. 


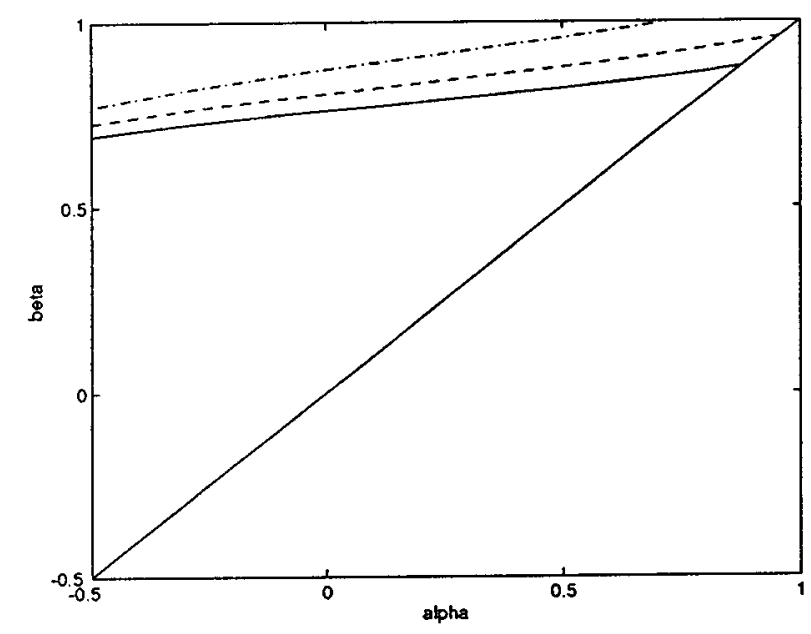

FiguRE 3.1. Positivity of quadrature weights for the Jacobi weight function $w^{(\alpha, \beta)}$

Conjecture 3.1. For the Jacobi weight function $w(t)=w^{(\alpha, \beta)}(t)$ the quadrature weights $\lambda_{i}$ and $\mu_{j}$ in (2.1), (2.2) are all positive if $(\alpha, \beta)$ is in the square $|\alpha| \leq \frac{1}{2}$, $|\beta| \leq \frac{1}{2}$.

The positivity expressed in Conjecture 3.1 has been proved in $\S 2$ at the four corner points of the square.

\section{REFERENCES}

1. A. Bellen, Alcuni problemi aperti sulla convergenza in media dell'interpolazione Lagrangiana estesa, Rend. Ist. Mat. Univ. Trieste 20 (1988), 1-9. MR 92e:41001

2. P. Erdös and P. Turán, On interpolation I, Ann. Math. 38 (1937), 142-155.

3. W. Gautschi, On mean convergence of extended Lagrange interpolation, J. Comput. Appl. Math. 43 (1992), 19-35. MR 93j:41003

4. W. Gautschi, Algorithm 726: ORTHPOL - A package of routines for generating orthogonal polynomials and Gauss-type quadrature rules, ACM Trans. Math. Software 20 (1994), 21-62.

5. W. Gautschi and S. Li, A set of orthogonal polynomials induced by a given orthogonal polynomial, Aequationes Math. 46 (1993), 174-198. MR 94e:33012

6. G. Pólya, Über die Konvergenz von Quadraturverfahren, Math. Z. 37 (1933), 264-286.

Department of Computer Sciences, Purdue University, West Lafayette, Indiana 47907-1398

E-mail address: wxg@cs.purdue.edu

Department of Mathematics, Southeastern Louisiana University, Hammond, Louisiana 70402

E-mail address: kli@selu.edu 